\title{
Role of myeloperoxidase in intracellular and extracellular chemiluminescence of neutrophils
}

\author{
HEATHER L NURCOMBE AND STEVEN W EDWARDS \\ From the Department of Biochemistry, University of Liverpool, Liverpool, UK
}

SUMMARY Activated polymorphonuclear leucocytes (neutrophils) can generate both intracellular and extracellular luminol dependent chemiluminescence. As luminol dependent chemiluminescence largely measures the activity of the myeloperoxidase- $\mathrm{H}_{2} \mathrm{O}_{2}$ system, and as the extracellular activity of this enzyme may be responsible for the tissue damage associated with inflammatory conditions such as rheumatoid arthritis, the aim of this work was to distinguish between intracellular and extracellular chemiluminescence so that the extracellular activity of this enzyme could be evaluated. Azide was used as a non-specific inhibitor of both intracellular and extracellular chemiluminescence, whereas anti-(human myeloperoxidase) IgG was used to inhibit specifically the extracellular activity of myeloperoxidase. Thus this IgG is a useful analytical tool for studying the extracellular activity of the myeloperoxidase $-\mathrm{H}_{2} \mathrm{O}_{2}$ system in the pathology of rheumatoid arthritis.

The production of a series of reactive oxidants by polymorphonuclear leucocytes (neutrophils) is necessary for the killing of certain types of microbial pathogens during infections. Two enzyme systems are responsible for the generation of a full complement of oxidants. Firstly, a plasma membrane bound NADPH oxidase ${ }^{1}$ generates $\mathrm{O}_{2}^{-}$and $\mathrm{H}_{2} \mathrm{O}_{2}$ during a respiratory burst which is activated during phagocytosis, ${ }^{2}$ and these may react together with a suitable transition metal salt to form a hydroxyl radical $(\cdot \mathrm{OH}) .^{3}$ Secondly, myeloperoxidase (a haemoprotein located within azurophilic granules) is discharged into the phagocytic vesicle by the process of degranulation and reacts with $\mathrm{H}_{2} \mathrm{O}_{2}$ (and possibly $\mathrm{O}_{2}^{-}$) to generate $\mathrm{HOCl}$ and related chloramines. The potency of $\cdot \mathrm{OH}$ and $\mathrm{HOCl}$ are well documented.

During phagocytosis of small particles such as bacteria these oxidants are generated and confined within phagolysosomes and few, if any, are released from the neutrophil. It is now known, however, that under certain conditions $\mathrm{O}_{2}^{-}, \mathrm{H}_{2} \mathrm{O}_{2}$, and granule enzymes can be secreted from neutrophils: in view of the high chemical reactivity of $\cdot \mathrm{OH}$ and $\mathrm{HOCl}$ such extracellular generation of oxidants may lead to damage to host tissues during inflammation. ${ }^{56}$

Accepted for publication 8 June 1988.

Correspondence to Dr Steven W Edwards, Department of Biochemistry, University of Liverpool, PO Box 147, Liverpool L693BX, UK.
As neutrophils are found in large numbers in the synovial fluid of patients with rheumatoid arthritis and have the potential to secrete reactive oxidants it has been proposed that the joint damage associated with this disease is attributable, at least in part, to neutrophil derived oxidants. ${ }^{7}$ There is, however, no direct evidence to support this proposal. In part this is because it is difficult to distinguish between intracellular and extracellular oxidant generation experimentally, and, also, the factors which both activate and regulate these processes in vivo are poorly understood.

Since the discovery that during the respiratory burst activated neutrophils also generate light $^{8}$ the technique of chemiluminescence has been widely used to measure the molecular controls that regulate reactive oxidant generation. The use of chemiluminescent probes such as luminol $^{9}$ greatly enhances the efficiency of photon detection, though the molecular species responsible for light emission have not yet been identified. Luminol dependent chemiluminescence is particularly useful for studies of neutrophil function as it measures, to a large extent, the activities of the NADPH oxidase and myeloperoxidase ${ }^{10}$ and is also capable of monitoring both intracellular and extracellular oxidant generation as luminol freely permeates these cells. Thus there have been several reports based on the use of neutrophil permeable or neutrophil impermeable non-specific scavenging agents which have ten- 
tatively distinguished between intracellular and extracellular events. ${ }^{11-15}$

We have recently shown that synovial fluid samples from patients with rheumatoid arthritis contain myeloperoxidase ${ }^{16}$ in a form which suggests that it has been actively secreted from neutrophils concomitant with the secretion of oxidants. ${ }^{17}$ The aim of the present study, therefore, was to establish methodologies to determine the extracellular activity of the myeloperoxidase- $\mathrm{H}_{2} \mathrm{O}_{2}$ system during neutrophil activation in order to evaluate the role of this enzyme in the pathology of this disease. As a specific inhibitor for myeloperoxidase does not exist $^{18}$ we used anti-(human myeloperoxidase) IgG to evaluate the extracellular activity of this enzyme.

\section{Materials and methods}

PREPARATION OF NEUTROPHILS

Neutrophils were prepared from $20 \mathrm{ml}$ heparinised venous blood from healthy volunteers using M-PRM (Flow Laboratories), exactly as described previously. ${ }^{16}$ After purification they were suspended in a buffer containing $(\mathrm{mM}): \mathrm{NaCl} 120 ; \mathrm{KCl} \mathrm{4.8}$; $\mathrm{KH}_{2} \mathrm{PO}_{4} 1 \cdot 2 ; \mathrm{CaCl}_{2} 1 \cdot 3 ; \mathrm{MgSO}_{4} 1 \cdot 2 ; \operatorname{HEPES}(\mathrm{N}-2$ hydroxyethylpiperazine- $N^{\prime}$-2-ethanesulphonic acid) $25(\mathrm{pH} 7 \cdot 4) ; 0.1 \%$ bovine serum albumin. Cells were counted after a suitable dilution in the above buffer with a Fuchs-Rosenthal haemocytometer slide and used within four hours of preparation. Neutrophils from 10 different donors were purified and assayed separately. Each assay was performed at least three times on neutrophils from the same donor.

\section{ANALYTICAL METHODS \\ Chemiluminescence}

Neutrophils were suspended in buffer containing $10 \mu \mathrm{M}$ luminol (5-amino-2,3-dihydro-1,4-phthalazenedione) at $10^{6}$ cells $/ \mathrm{ml}$ in a total volume of $1 \mathrm{ml},{ }^{10}$ and chemiluminescence was measured with an LKB Wallac 1250 luminometer.

\section{$\mathrm{O}_{2}^{-}$and $\mathrm{H}_{2} \mathrm{O}_{2}$ generation}

$\mathrm{O}_{2}^{-}$generation was measured in a continuous assay by monitoring the rate of reduction of ferricytochrome $c .{ }^{19}$ The assay, total volume of $1 \mathrm{ml}$, was performed in a Perkin-Elmer lambda 5 spectrophotometer and contained $75 \mu \mathrm{M}$ cytochrome $c$ plus $5 \times 10^{5}$ cells. $\mathrm{H}_{2} \mathrm{O}_{2}$ generation was measured by a fluorimetric assay ${ }^{20}$ in a $2.5 \mathrm{ml}$ reaction mixture containing $4 \mu \mathrm{M}$ scopoletin, $5 \mu \mathrm{g} / \mathrm{ml}$ horseradish peroxidase, and $2.5 \times 10^{6}$ neutrophils.

\section{Other methods}

Human myeloperoxidase was prepared from buffy coats by the method described by Pember $e t a l^{21}$ and used to raise rabbit anti-(human myeloperoxidase) antiserum, ${ }^{18}$ from which a purified IgG fraction was isolated. ${ }^{22}$

\section{Results}

KINETICS OF CHEMILUMINESCENCE, $\mathrm{O}_{2}^{-}$AND $\mathrm{H}_{2} \mathrm{O}_{2}$ PRODUCTION

When neutrophil suspensions were stimulated by the addition of the chemotactic peptide fMet-LeuPhe plus cytochalasin B the rate of chemiluminescence reached an initial peak within one minute of addition of stimulus (Fig. 1a). After this time the rate declined and was followed by a second, more sustained, maximum. The maximal rates of $\mathrm{O}_{2}^{-}$ and $\mathrm{H}_{2} \mathrm{O}_{2}$ production observed coincided with the initial increase in chemiluminescence-that is, within one minute- but during the second phase of chemiluminescence the rates of $\mathrm{O}_{2}^{-}$and $\mathrm{H}_{2} \mathrm{O}_{2}$ generation were barely detectable above background levels (Fig. 1b). When neutrophils were stimulated by fMet-Leu-Phe in the absence of
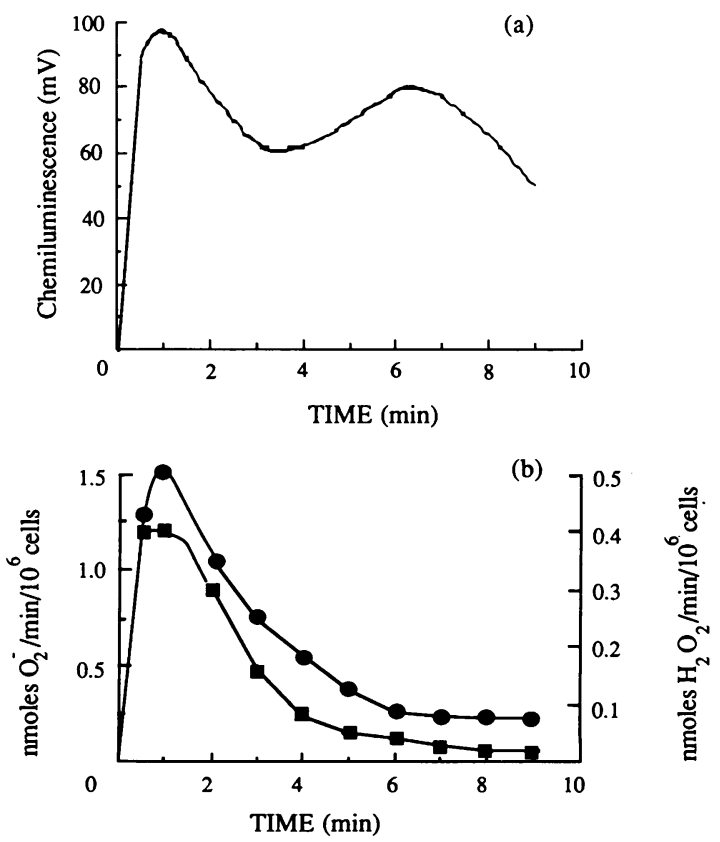

Fig. 1 Effect of fMet-Leu-Phe plus cytochalasin B on chemiluminescence, $\mathrm{O}_{2}$ and $\mathrm{H}_{2} \mathrm{O}_{2}$ production. Neutrophils were incubated at $37^{\circ} \mathrm{C}$ as described in 'Materials and methods' and at time zero $1 \mu \mathrm{M}$ fMet-Leu-Phe plus $1 \mu \mathrm{g} / \mathrm{ml}$ cytochalasin $B$ (final concentrations) was added.

(a) Chemiluminescence response; (b) rates of $\mathrm{O}_{2}^{-}\left(\mathrm{O}^{-}\right.$and $\mathrm{H}_{2} \mathrm{O}_{2}(\square)$ production. 
cytochalasin B a similar but lower magnitude chemiluminescence profile was observed (Fig. 2a), and, again, rates of $\mathrm{O}_{2}^{-}$and $\mathrm{H}_{2} \mathrm{O}_{2}$ generation were only detectable within the first few minutes of addition of stimulus (Fig. 2b). Upon addition of the protein kinase $c$ activator phorbol myristate acetate (PMA) the rate of chemiluminescence reached a maximal value within six to eight minutes (Fig. 3a), and the rates of $\mathrm{O}_{2}^{-}$and $\mathrm{H}_{2} \mathrm{O}_{2}$ generation followed a similar time course (Fig. $3 b$ ).

Thus particularly after activation with fMet-LeuPhe (in the presence or absence of cytochalasin B) rates of chemiluminescence from three to eight minutes after stimulation do not parallel rates of $\mathrm{O}_{2}^{-}$ and $\mathrm{H}_{2} \mathrm{O}_{2}$ generation. This may indicate that an $\mathrm{O}_{2}^{-}$ or $\mathrm{H}_{2} \mathrm{O}_{2}$ independent oxidant is responsible for photon emission. Alternatively, as the substrates used for the detection of $\mathrm{O}_{2}^{-}$and $\mathrm{H}_{2} \mathrm{O}_{2}$ generation cannot penetrate neutrophils this may suggest that oxidant generation occurs extracellularly in the first instance (during the initial one to two minutes after stimulation) and then later occurs intracellularly.
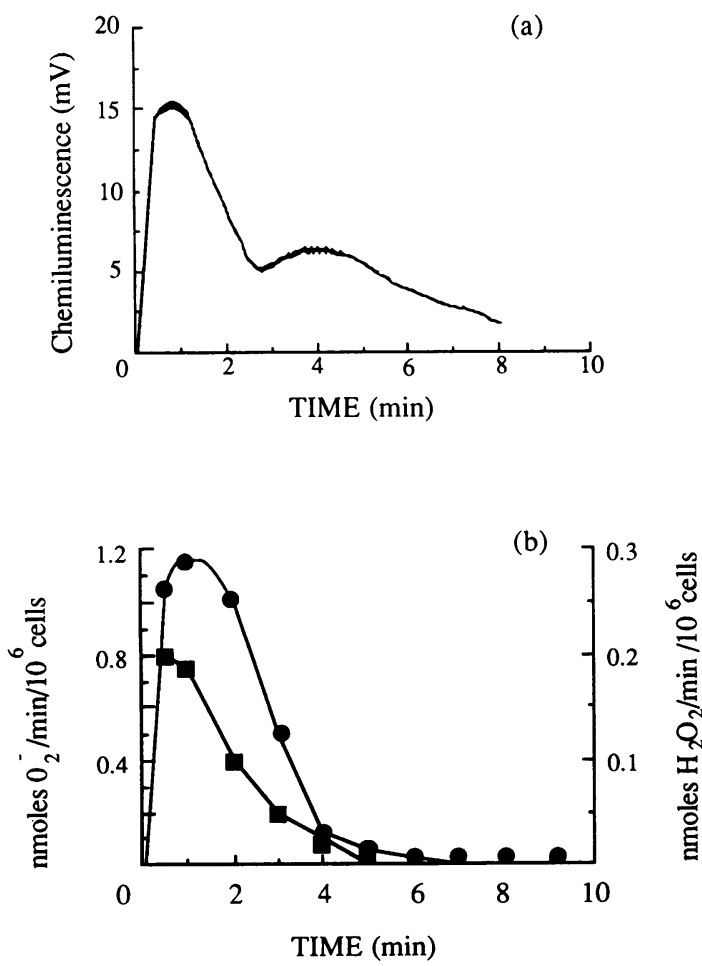

Fig. 2 Effect of fMet-Leu-Phe on chemiluminescence, $\mathrm{O}_{2}^{-}$ and $\mathrm{H}_{2} \mathrm{O}_{2}$ production. Details were as described in the legend to Fig. 1 except that at time zero the suspensions were stimulated by the addition of $1 \mu M \mathrm{fMet}$-Leu-Phe (final concentration).
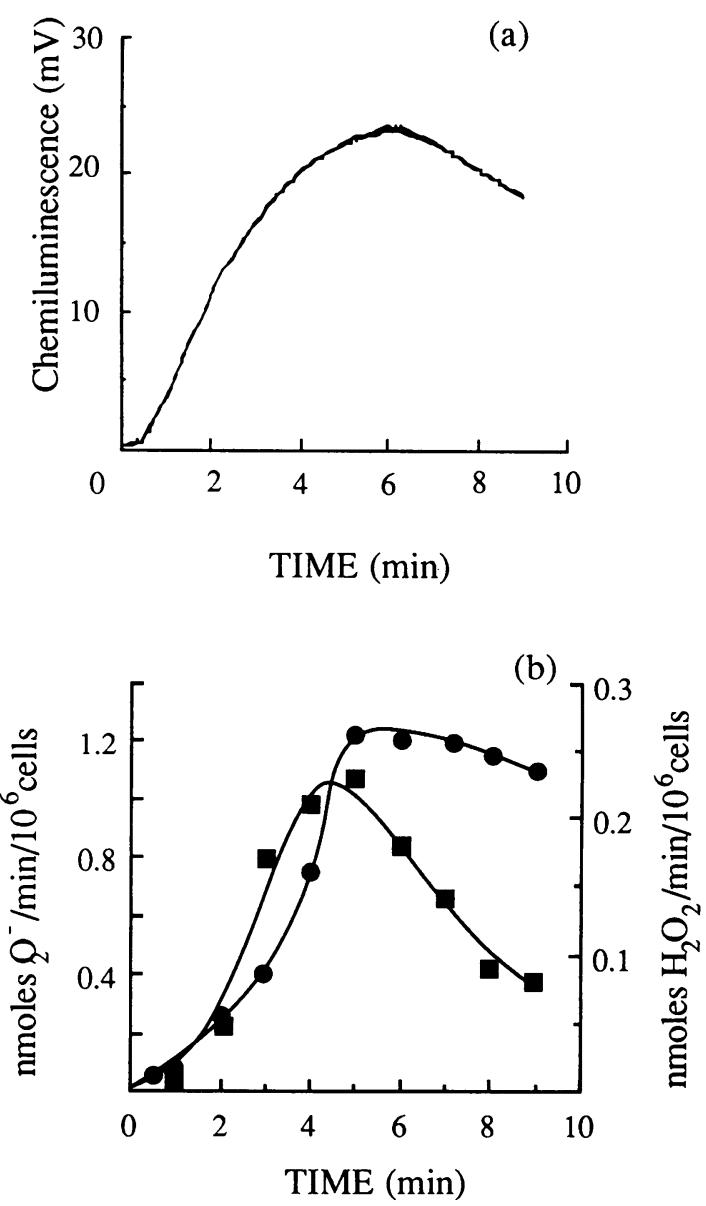

Fig. 3 Effect of phorbol myristate acetate (PMA) on chemiluminescence, $\mathrm{O}_{2}^{-}$and $\mathrm{H}_{2} \mathrm{O}_{2}$ production. Details were as described in the legend to Fig. 1 except that at time zero suspensions were stimulated by the addition of $0 \cdot 1 \mathrm{\mu g} / \mathrm{ml}$ PMA (final concentration).

Enhancement of chemiluminescence by exogenous myeloperoxidase

As luminol dependent chemiluminescence largely requires both the generation of oxidants and degranulation of myeloperoxidase ${ }^{10}$ the effect of adding exogenous, purified myeloperoxidase to fMet-LeuPhe and PMA stimulated neutrophil suspensions was investigated. When exogenous myeloperoxidase was added to PMA stimulated suspensions at various time intervals after activation the rate of chemiluminescence was dramatically enhanced (Fig. $4 a)$. The potentiating effect varied with the time interval between the addition of myeloperoxidase and the addition of PMA. For example, when 
myeloperoxidase was added three to six minutes after the addition of PMA a much greater rate of chemiluminescence was observed than when it was added 20 minutes after PMA. When added three minutes after PMA myeloperoxidase increased the rate of chemiluminescence 13 -fold, whereas when added between eight and 20 minutes after PMA the rate was only enhanced about fivefold-that is, the potentiating effect of myeloperoxidase added after three minutes was $2 \cdot 5$-fold (SD $0 \cdot 25, n=6$ ) greater than that observed when added 8-20 minutes after stimulation.
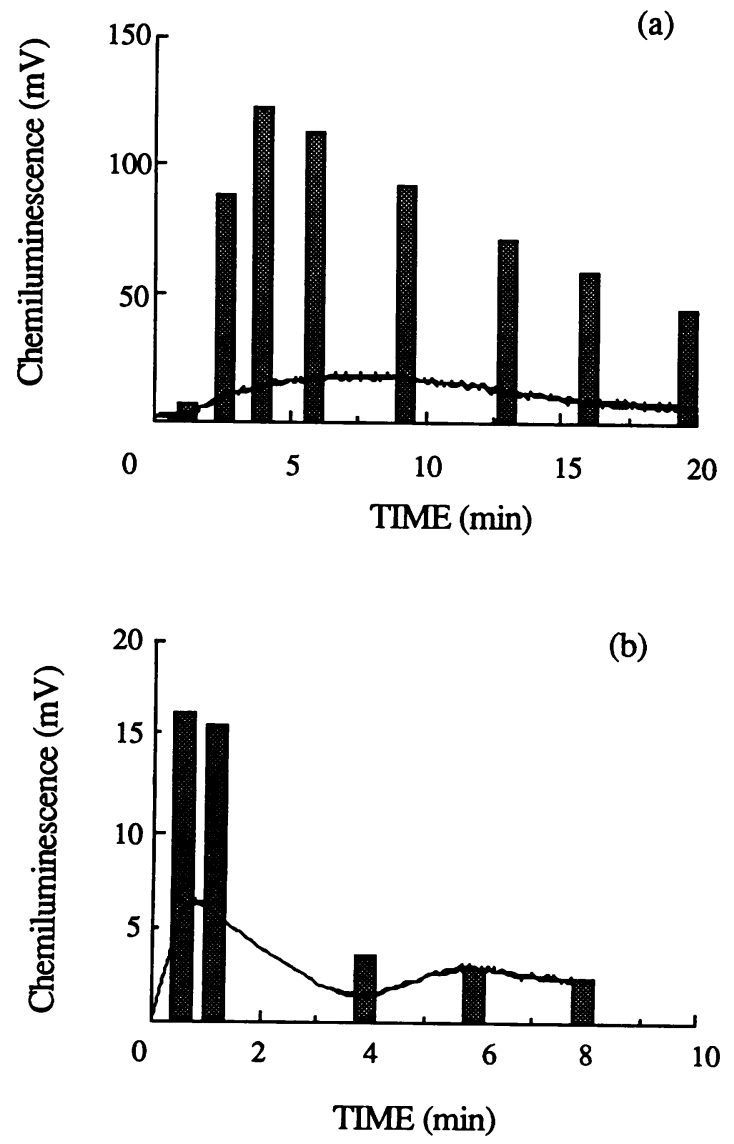

Fig. 4 Effect of exogenously added myeloperoxidase on neutrophil chemiluminescence. Neutrophil suspensions were stimulated by the additions of (a) $0 \cdot 1 \mu \mathrm{g} / \mathrm{ml}$ phorbol myristate acetate or (b) $1 \mu \mathrm{M}$ fMet-Leu-Phe and the chemiluminescence responses measured (solid traces). In a number of separate incubations the suspensions were stimulated as described and then at various time intervals after stimulation $2 \mu \mathrm{g}$ of purified myeloperoxidase was added and its effect on the chemiluminescence response measured (hatched bars).

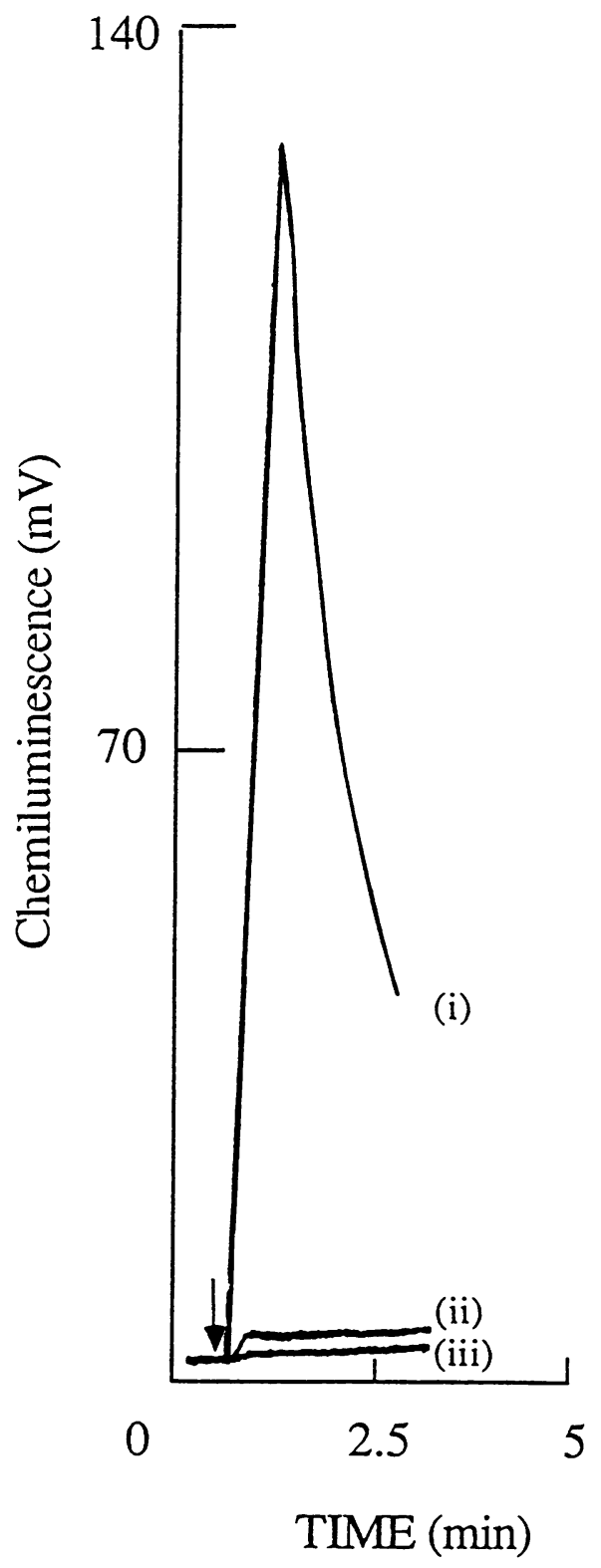

Fig. 5 Effect of azide and anti-(human myeloperoxidase) IgG on myeloperoxidase- $\mathrm{H}_{2} \mathrm{O}_{2}$ chemiluminescence. Purified myeloperoxidase (1 $\mu \mathrm{g})$ was incubated at $37^{\circ} \mathrm{C}$ in buffer containing $10 \mu \mathrm{M}$ luminol. At the time indicated by the arrow $60 \mu M \mathrm{H}_{2} \mathrm{O}_{2}$ (final concentration) was added and the chemiluminescence response measured. (i) No additions; (ii) myeloperoxidase incubated with $150 \mu \mathrm{g}$ of anti-(human myeloperoxidase) IgG for five minutes before addition of $\mathrm{H}_{2} \mathrm{O}_{2}$; (iii) chemiluminescence response obtained when $1 \mathrm{mM}$ azide was added to the purified myeloperoxidase. 


\section{Nurcombe, Edwards}

Similarly, a time dependent enhancement of fMet-Leu-Phe stimulated chemiluminescence by exogenous myeloperoxidase was observed (Fig. 4b). Maximal enhancement of chemiluminescence was found when myeloperoxidase was added about one minute after fMet-Leu-Phe (coinciding with the time of maximal generation of extracellular $\mathrm{O}_{2}^{-}$and $\mathrm{H}_{2} \mathrm{O}_{2}$, Fig. 2b), but when added after this time little, if any, potentiating effect was observed. Thus when myeloperoxidase was added one minute after fMetLeu-Phe the potentiating effect was $2 \cdot 4$-fold (SD $0 \cdot 6, \mathrm{n}=4)$ greater than when added five minutes after stimulation.

As exogenous myeloperoxidase cannot penetrate neutrophils the enhanced chemiluminescence indicates that $\mathrm{H}_{2} \mathrm{O}_{2}$ (and presumably $\mathrm{O}_{2}^{-}$) are present in the extracellular medium. Thus during the initial

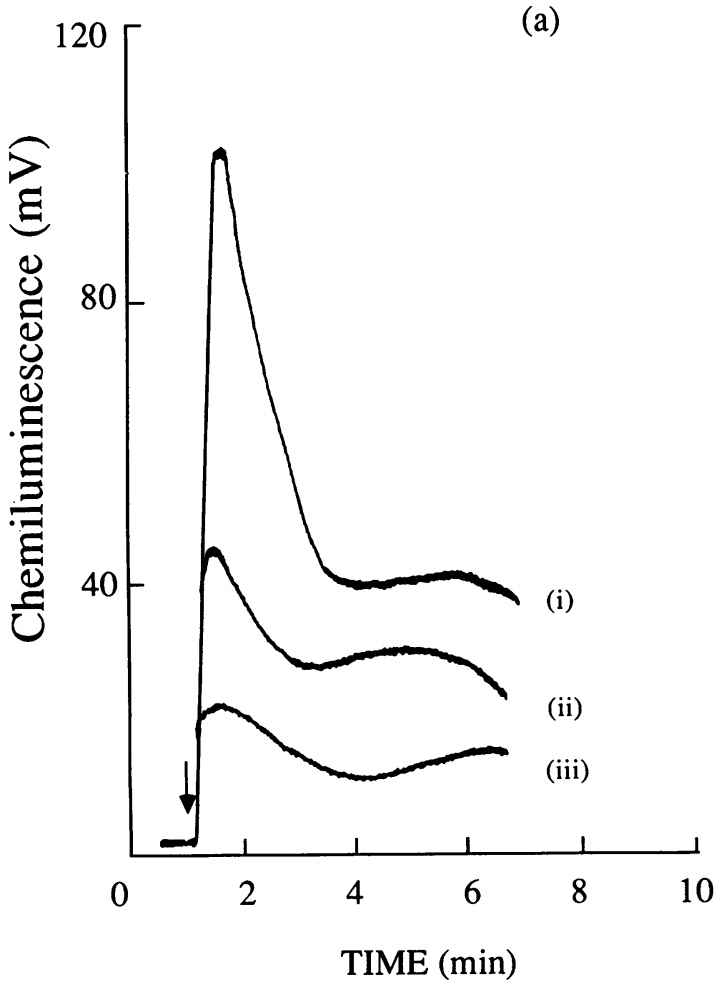

Fig. 6 Effect of azide and anti-(human myeloperoxidase) IgG on neutrophil chemiluminescence. Neutrophil suspensions were incubated as described in 'Materials and methods' in the absence (i) or presence of $150 \mu \mathrm{g} / \mathrm{ml} \mathrm{IgG}$ (ii) or $1 \mathrm{mM}$ azide (iii). At the time indicated by the arrow suspensions were stimulated by the additions of

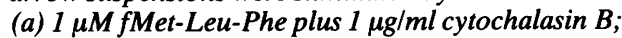
(b) $1 \mu M$ fMet-Leu-Phe; or (c) $0.1 \mu \mathrm{g} / \mathrm{ml}$ phorbol myristate acetate. periods of both PMA and fMet-Leu-Phe stimulation, when exogenous myeloperoxidase has its greatest effect, higher levels of $\mathrm{H}_{2} \mathrm{O}_{2}$ (and $\mathrm{O}_{2}^{-}$) must be present extracellularly than at those times when exogenous myeloperoxidase has a lower effect.

Inhibition of myeloperoxidase by azide and anti(human myeloperoxidase) IgG

Although azide is commonly used as an inhibitor of myeloperoxidase, it has limited usefulness in these studies as it is a non-specific inhibitor of many proteins containing haem and can also quench singlet $\mathrm{O}_{2}^{-}$and $\cdot \mathrm{OH}^{3}$ It is capable, however, of
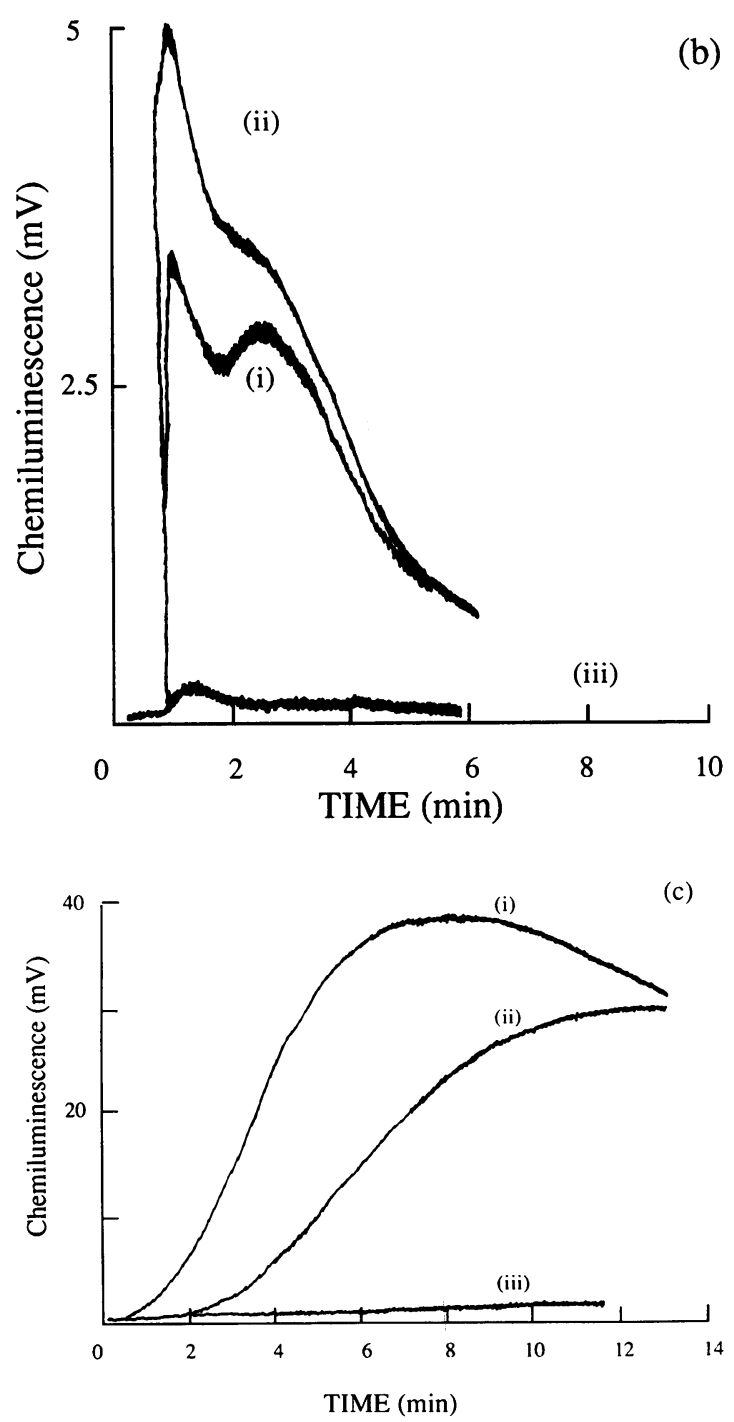
inhibiting both intracellular and extracellular events. We have previously used antiserum raised to purified myeloperoxidase as a specific inhibitor of this enzyme in order to determine its role in the regulation of the respiratory burst. ${ }^{18}$ Therefore an IgG fraction prepared from this antiserum was tested for its ability to inhibit myeloperoxidase dependent chemiluminescence. Figure 5 shows that the chemiluminescence of a cell free system comprising purified myeloperoxidase and $\mathrm{H}_{2} \mathrm{O}_{2}$ was almost completely inhibited by both azide and the anti-(human myeloperoxidase) IgG. As the IgG cannot penetrate neutrophils this agent should therefore only inhibit neutrophil chemiluminescence due to the activity of an extracellular myeloperoxidase- $\mathrm{H}_{2} \mathrm{O}_{2}$ system. Azide, on the other hand, will inhibit both intracellular and extracellular events.

\section{Effect of azide and anti-(human myeloperoxidase) IgG on neutrophil chemiluminescence}

The addition of $1 \mathrm{mM}$ azide greatly reduced the chemiluminescence response activated by the addition of fMet-Leu-Phe plus cytochalasin B to neutrophils, with inhibition at one and five minutes of $78 \%($ SD $8 \%, n=4)$ and $75 \%($ SD $8 \%, n=4)$ respectively (Fig. 6a). Similarly, anti-(myeloperoxidase) IgG also inhibited this chemiluminescence response, but in contrast with the effect of azide inhibited the response at one and 5 minutes by only $59 \%($ SD $1 \%, n=4)$ and $27 \%($ SD $5 \%, n=4)$ respectively. This observation is consistent with the idea that azide, which freely penetrates neutrophils, inhibits equally both the intracellular and extracellular chemiluminescence due to myeloperoxidase, whereas the IgG, which cannot penetrate the neutrophil, only inhibits the extracellular myeloperoxidase activity. Thus the chemiluminescence observed one minute after the addition of this stimulus is largely due to the extracellular activity of the myeloperoxidase- $\mathrm{H}_{2} \mathrm{O}_{2}$ system.

In contrast with azide, IgG had very little inhibitory effect on the initial chemiluminescence response activated by fMet-Leu-Phe alone, and even potentiated the response (Fig. 6b). These results strongly suggest that the initial (one minute) peak of chemiluminescence activated by fMet-Leu-Phe alone is not due to extracellular myeloperoxidase activity.

Whereas azide greatly reduced the rate of PMA stimulated chemiluminescence, IgG only reduced the initial rate. For example, IgG inhibited the chemiluminescence at five minutes by $43 \%$ (SD $20 \%, n=4)$ and that at 13 minutes by only $7 \%$ (SD $2 \%, n=4$ ) (Fig. 6c). These data are consistent with the idea that the extracellular activity of myeloperoxidase is maximal three to six minutes after stimulation by PMA, whereas from about 10 minutes after stimulation by this agent the intracellular activity of this enzyme predominates.

\section{Discussion}

It is now appreciated that in addition to the generation of reactive oxidants within phagolysosomes during phagocytosis of bacteria, neutrophils also have the capacity to secrete these oxidants. It is currently believed that the two most toxic species are $\cdot \mathrm{OH}$ and $\mathrm{HOCl}$ and oxidants formed either directly or indirectly from these. ${ }^{3}$ As the generation of $\mathrm{OH}$ requires a transition metal in a suitable form $^{7}$ it has been proposed that the chelation of transition metals by compounds such as desferrioxamine may reduce joint damage in rheumatoid arthritis owing to the production of this species. ${ }^{23-25}$ The chelation of transition metals, however, will not inhibit the activity of the myeloperoxidase $-\mathrm{H}_{2} \mathrm{O}_{2}$ system, and it is known that this enzyme may also be secreted from neutrophils and this may also contribute to tissue damage. Therefore before the role of neutrophil derived oxidants in the pathology of rheumatoid arthritis can be evaluated it is necessary (a) to determine the physiological conditions which result in the secretion of oxidants from activated neutrophils; (b) to develop assays to distinguish between intracellular and extracellular production; and $(c)$ to measure the extracellular activity of the myeloperoxidase- $\mathrm{H}_{2} \mathrm{O}_{2}$ system. The experiments described in this report were designed to solve some of these problems.

When neutrophil suspensions were stimulated with either the chemotactic peptide, fMet-Leu-Phe, (in the presence or absence of cytochalasin B) or PMA, both intracellular and extracellular oxidant generation was detected, though with the latter stimulus the distinction was not as well defined. These conclusions were based on the facts that $(a)$ in the assays to determine the rates of $\mathrm{O}_{2}^{-}$and $\mathrm{H}_{2} \mathrm{O}_{2}$ generation the substrates used do not penetrate the cells and therefore only extracellular oxidants are measured (Figs 1-3); (b) exogenously added myeloperoxidase could cause preferential enhancement of chemiluminescence depending upon the time that it was added after stimulation (Fig. 4); and (c) anti(human myeloperoxidase) IgG could inhibit chemiluminescence at certain times after stimulation but not others (Fig. 6).

The combined use of azide and anti-(human myeloperoxidase) IgG to inhibit the total cellular activity or just extracellular myeloperoxidase activity, respectively, provides a novel approach to delineating the contribution of the released enzyme during neutrophil activation. The IgG was as effec- 
tive as azide in inhibiting the chemiluminescence of a cell free system comprising purified myeloperoxidase and $\mathrm{H}_{2} \mathrm{O}_{2}$ (Fig. 5), and because it is membrane impermeable it specifically inhibited chemiluminescence due to extracellular myeloperoxidase. This approach showed that the initial chemiluminescence responses of both the fMet-Leu-Phe plus cytochalasin B and PMA stimulated suspensions was due, at least in part, to extracellular myeloperoxidase. Substantial portions of both fMet-Leu-Phe and PMA simulated chemiluminescence were not inhibited by either azide or IgG, however, suggesting that these stimuli activated considerable myeloperoxidase independent, luminol dependent chemiluminescence. Further work is clearly necessary to identify the molecular species responsible for this photon emission and to determine whether this phenomenon is related to lucigenin dependent chemiluminescence, which also appears to be independent of myeloperoxidase. ${ }^{10}$

Several investigators have shown that synovial fluid contains factors which can activate neutrophil oxidant generation. ${ }^{26}$ In view of the fact that we have proposed a role for extracellular myeloperoxidase in the pathology of rheumatoid arthritis ${ }^{16}$ it will now be possible to extend the approach described here to distinguish clearly between intracellular and extracellular oxidant generation by synovial fluid neutrophils and also to assess the role of released myeloperoxidase. This work thus provides the basis for evaluating the role of neutrophil derived oxidants in the joint damage and destruction associated with this disease.

We thank the Arthritis and Rheumatism Council for generous financial support.

\section{References}

1 Rossi $\mathrm{F}$. The $\mathrm{O}_{2}$-forming oxidase of the phagocytes: nature, mechanisms of activation and function. Biochim Biophys Acta 1986; 853: 65-89.

2 Babior B M. Oxidants from phagocytes: agents of defense and destruction. Blood 1984; 64: 959-66.

3 Halliwell B, Gutteridge J M C. Free radicals in biology and medicine. Oxford: Clarendon Press, 1985: 346.

4 Klebanoff S J, Clark R A. The neutrophil: function and clinical disorders. Amsterdam: North-Holland, 1978: 810.

5 Fantone J C, Ward P A. Role of oxygen-derived free radicals and metabolites in leukocyte-dependent inflammatory reactions. Am J Pathol 1982; 107: 397-418.

6 Torrielli M V, Dianzani M U. Free radicals in inflammatory disease. In: Armstrong D, Sohal R S, Cutler R G, Slater T F, eds. Free radicals in molecular biology, aging and disease. New York: Raven Press, 1984: 355-79.

7 Halliwell B, Gutteridge J M C, Blake D. Metal ions and oxygen radical reactions in human inflammatory joint disease. Philos Trans $R$ Soc Lond [Biol] 1985; 311: 659-71.

8 Allen R C, Stjeinholm R L, Steele R H. Evidence for the generation of an electronic excitation state(s) in human polymorphonuclear leukocytes and its participation in bacterial killing. Biochem Biophys Res Commun 1972; 47: 679-84.

9 Allen R C, Loose L D. Phagocytic activation of a luminoldependent chemiluminescence in rabbit alveolar and peritoneal macrophages. Biochem Biophys Res Commun 1976; 96: 245-52.

10 Edwards S W. Luminol- and lucigenin-dependent chemiluminescence of neutrophils: role of degranulation. J Clin Lab Immunol 1987; 22: 35-9.

11 Bender J G, Van Epps D E. Analysis of the bimodal chemiluminescence pattern stimulated in human neutrophils by chemotactic peptide. Infect Immun 1983; 41: 1062-70.

12 Hallett M B, Campbell A K. Two distinct mechanisms for stimulation of oxygen-radical production by polymorphonuclear leukocytes. Biochem J 1983; 216: 459-65.

13 Briheim G, Stendahl O, Dahlgren C. Intra- and extracellular events in luminol-dependent chemiluminescence of polymorphonuclear leukocytes. Infect Immun 1984; 45: 1-5.

14 Dahlgren C, Aniansson H, Magnusson K-E. Pattern of formylmethionyl-leucyl-phenylalanine-induced luminol- and lucigenin-dependent chemiluminescence in human neutrophils. Infect Immun 1985; 47: 326-8.

15 Dahlgren $C$. Difference in extracellular radical release after chemotactic factor and calcium ionophore activation of the oxygen radical generating system in human neutrophils. Biochim Biophys Acta 1987; 930: 33-8.

16 Edwards S W, Hughes V, Barlow J, Bucknall R. Immunological detection of myeloperoxidase in synovial fluid from patients with rheumatoid arthritis. Biochem $J$ 1988; 250: 81-5.

17 Edwards S W, Nurcombe H L, Hart C A. Oxidative inactivation of myeloperoxidase released from human neutrophils. Biochem J 1987; 245: 925-8.

18 Edwards S W, Swan T F. Regulation of superoxide generation by myeloperoxidase during the respiratory burst of human neutrophils. Biochem $J$ 1986; 237: 601-4.

19 Babior B M, Kipnes R S, Curnutte J T. Biological defense mechanisms. The production by leukocytes of superoxide, a potential bacteriocidal agent. J Clin Invest 1973; 52: 741-4.

20 Root R K, Metcalf J, Oshino N, Chance B. $\mathrm{H}_{2} \mathrm{O}_{2}$ release from human granulocytes during phagocytosis. i. Documentation, quantitation and some regulating factors. J Clin Invest 1975; 55: 945-5.

21 Pember S O, Shapira R, Kinkade J M Jr. Multiple forms of myeloperoxidase from human neutrophilic granulocytes: evidence for differences in compartmentalization, enzymatic activity and sub-unit structure. Arch Biochem Biophys 1983; 221: 391-403.

22 Hurn B A L, Chantler S M. Production of reagent antibodies. Methods Enzymol 1980; 70: 104-42.

23 Blake D R, Winyard P, Lunec J, et al. Cerebral and ocular toxicity induced by desferrioxamine. $Q J$ Med 1985; 56: 345-55.

24 Polson R J, Jawad A, Bomford A, Berry H, Williams R. Treatment of rheumatoid arthritis with desferrioxamine: relation between stores of iron before treatment and side effects. $\mathrm{Br}$ Med $J$ 1985; 291: 448.

25 Polson R J, Jawad A, Bomford A, Berry H, Williams R. Treatment of rheumatoid arthritis with desferrioxamine. $Q J$ Med 1986; 61: 1153-8.

26 Gale R, Bertouch J V, Bradley J, Roberts-Thomson P J. Direct activation of neutrophil chemiluminescence by rheumatoid sera and synovial fluid. Ann Rheum Dis 1983; 42: 158-62. 